\title{
INVARIANT AFFINE CONNECTIONS ON SYMMETRIC SPACES
}

\author{
H. TURNER LAQUER
}

(Communicated by Jonathan M. Rosenberg)

\begin{abstract}
The space of left invariant affine connections is determined for the compact irreducible Riemannian symmetric spaces.
\end{abstract}

\section{INTRODUCTION}

The objective of this paper is to describe the space of invariant affine connections on arbitrary compact irreducible Riemannian symmetric spaces. It represents a sequel to [8] where the bi-invariant affine connections on compact Lie groups were determined. The main result, Theorem 2.1 , shows that with few exceptions the only invariant affine connection is the canonical connection.

The proof of the main result is split into two cases. The Hermitian symmetric case, considered in $\S 4$, results in an even stronger uniqueness theorem for a wide class of bundles (see Theorem 4.1). The classification in the non-Hermitian case is based on a case by case study using representation theory. Casimir operators (§3) provide a key computational tool. Section 5 gives details about the main computation and $\S 6$ gives a more precise description of the new invariant affine connections on the spaces $S U(n) / S O(n)$ and $S U(2 n) / S P(n)$.

\section{INVARIANT AFFINE CONNECTIONS ON HOMOGENEOUS SPACES}

A homogeneous principal bundle is a principal bundle over a homogeneous space $G / H$ that has a $G$-action on the total space that is compatible with the given action on the base manifold. Up to isomorphism in a natural category $[7,8]$, such bundles are given by constructions of the form

$$
P_{\lambda}=G \times_{H} U=G \times U /(g h, u) \sim(g, \lambda(h) u) .
$$

This principal $U$-bundle over the homogeneous space $G / H$ is defined by means of a structure homomorphism $\lambda: H \rightarrow U$. The equation $h \cdot p_{0}=p_{0} \cdot \lambda(h)$ defines this homomorphism canonically from the basepoint $p_{0}=[e, e]$.

Wang's theorem [11] describes the space of invariant connections in a homogeneous principal bundle. Specifically, let $\mathfrak{g}, \mathfrak{h}$, and $\mathfrak{u}$ be the Lie algebras of $G, H$, and $U$ respectively, and let $T \lambda: \mathfrak{h} \rightarrow \mathfrak{u}$ be the differential of $\lambda$. Then the invariant connections in $P_{\lambda}$ are described by linear mappings $\xi: \mathfrak{g} \rightarrow \mathfrak{u}$

Received by the editors August 2, 1990.

1991 Mathematics Subject Classification. Primary 53C05; Secondary 53C35, 32M15.

Key words and phrases. Affine, connection, invariant, symmetric space. 
satisfying $\xi(x)=T \lambda(x) \quad \forall x \in \mathfrak{h}$ and $\xi\left(\operatorname{Ad}_{h} x\right)=\operatorname{Ad}_{\lambda(h)} \xi(x) \quad \forall x \in \mathfrak{g}, h \in H$ When the homogeneous space is reductive, i.e., when the Lie algebra $\mathfrak{g}$ splits into a direct sum $\mathfrak{h} \oplus \mathfrak{m}$ with $\operatorname{Ad}(H) \mathfrak{m} \subseteq \mathfrak{m}$, such connections are given by linear mappings $\xi: \mathfrak{m} \rightarrow \mathfrak{u}$ satisfying $\xi\left(\operatorname{Ad}_{h} x\right)=\operatorname{Ad}_{\lambda(h)} \xi(x) \quad \forall x \in \mathfrak{m}, h \in H$. Reductivity, which is automatic if $H$ is compact or semisimple, will be assumed throughout this paper.

An invariant affine connection on a homogeneous space $G / H$ is a connection in the frame bundle that is also $G$-invariant. By viewing a frame at a point $m \in G / H$ as an isomorphism $p: \mathfrak{m} \rightarrow T_{m}(G / H)$ and by letting the base point be the canonical projection $\mathfrak{m} \rightarrow T_{e H}(G / H)$, the structure homomorphism becomes Ad: $H \rightarrow \operatorname{Aut}(\mathfrak{m})$. By Wang's theorem, the space of invariant affine connections consists of all linear mappings $\xi: \mathfrak{m} \rightarrow \operatorname{End}(\mathfrak{m})$ satisfying $\xi\left(\operatorname{Ad}_{h} x\right)=$ $\operatorname{Ad}_{h} \circ \xi(x) \circ \operatorname{Ad}_{h^{-1}} \quad \forall x \in \mathfrak{m}, h \in H$. The identification $\xi(x)(y)=\eta(x, y)$ shows that this is equivalent to the set of all bilinear mappings $\eta: \mathfrak{m} \times \mathfrak{m} \rightarrow \mathfrak{m}$ satisfying $\eta\left(\operatorname{Ad}_{h} x, \operatorname{Ad}_{h} y\right)=\operatorname{Ad}_{h} \eta(x, y) \quad \forall x, y \in \mathfrak{m}, h \in H$. Finally, the universal mapping property of tensor product shows that this is equivalent to the space $\operatorname{Hom}_{H}^{\mathbb{R}}(\mathfrak{m} \otimes \mathfrak{m}, \mathfrak{m})$ of intertwining maps $\mathfrak{m} \otimes \mathfrak{m} \rightarrow \mathfrak{m}$ (cf. $\left.[8,10]\right)$.

\section{INVARIANT AFFINE CONNECTIONS ON SYMMETRIC SPACES}

A symmetric space is a triple $(G, H, \sigma)$ where $\sigma$ is an involutive automorphism of $G$ and $H$ is a closed subgroup of $G$ lying between the fixed point subgroup of $\sigma$ and the identity component thereof. A symmetric space has a canonical reductive splitting $\mathfrak{g}=\mathfrak{h} \oplus \mathfrak{m}$ defined by letting $\mathfrak{h}$ and $\mathfrak{m}$ be the +1 and -1 eigenspaces of the differential $T \sigma: \mathfrak{g} \rightarrow \mathfrak{g}$ respectively. Standard classification results [4] divide the simply connected compact irreducible Riemannian symmetric spaces into two classes: type $I$ in which $G$ is simple and type II in which $G$ is the product of two copies of a simple Lie group which are interchanged by the automorphism $\sigma$. Invariant affine connections for type II symmetric spaces correspond to the bi-invariant affine connections on simple Lie groups which were considered in [8]. The main objective of this paper is the following theorem, the proof of which will be delayed to $\S 5$.

Theorem 2.1. The set of invariant affine connections on type I symmetric spaces consists of just the canonical connection $(\eta=0)$ in all cases except for the following:

$$
\begin{array}{lcl}
\text { AI } & S U(n) / S O(n) & n \geq 3, \\
\text { AII } & S U(2 n) / S P(n) & n \geq 3, \\
\text { EIV } & E_{6} / F_{4} . &
\end{array}
$$

Each of these spaces has a one-dimensional family of invariant affine connections. Note, $S O(6) / S O(3) \times S O(3)$ behaves like $S U(4) / S O(4)$.

\section{CASIMIR OPERATORS}

Given a representation $\rho: H \rightarrow$ Aut $(V)$, the Casimir operator of $V$ is defined by $\Gamma(V)=\sum\left(T \rho\left(e_{j}\right)\right)^{2}$ where $\left\{e_{j}\right\}$ is an orthonormal basis for the Lie algebra $\mathfrak{h}$. This depends on the choice of an inner product on $\mathfrak{h}$ and in the context of type I symmetric spaces, the natural inner product is given by the 
restriction to $\mathfrak{h}$ of $-B_{\mathfrak{g}}$ (the negative of the Killing form on $\mathfrak{g}$ ). If $V$ is irreducible then $\Gamma(V)$ is a negative or zero scalar. In particular, for symmetric spaces the Casimir operator of the $H$-module $\mathfrak{m}$ is $-\frac{1}{2}$ [6].

The Lie algebra $\mathfrak{h}$ splits into a direct sum $\mathfrak{z} \oplus\left(\bigoplus_{j=1}^{p} \mathfrak{h}_{j}\right)$ of $H$-modules, where $\mathfrak{z}$ is the center of $\mathfrak{h}$ and the $\mathfrak{h}_{j}$ are the simple ideals in $\mathfrak{h}$. In all the type $I$ symmetric spaces the center is, at worst, one-dimensional. This allows complex irreducible representations of $H$ to be expressed as $V=\left(k \cdot \beta, \Lambda_{1}, \ldots, \Lambda_{p}\right)$. This notation indicates that $V$ is the tensor product of a one-dimensional representation $(k \cdot \beta)$ of the center together with the representation of highest weight $\left(\Lambda_{j}\right)$ for each $H_{j}$. At the group level, complex representations of $S^{1}$ are parameterized by the integers. The problem at the algebra level is to know which representation corresponds to the integer 1 . The point of this notation is to force the $H$-module $\mathfrak{m}$ to have $z$-part $( \pm 1 \cdot \beta)$. The nontriviality of $(\beta)$ can be proven from Lemma 3.1 and computation of Casimir operators.

The Casimir operator can be expressed in terms of indices of representations [6]. In this context,

$$
\begin{aligned}
\Gamma\left(k \cdot \beta, \Lambda_{1}, \ldots, \Lambda_{p}\right) & =-\gamma_{0} \cdot k^{2}-\sum_{j=1}^{p} \frac{\operatorname{dim}\left(\mathfrak{h}_{j}\right) \cdot L(G)}{2 \cdot I\left(\mathfrak{h}_{j}\right)} \cdot \frac{K\left(\Lambda_{j}\right)}{\operatorname{dim}\left(\Lambda_{j}\right)} \\
& =-\gamma_{0} \cdot k^{2}-\sum_{j=1}^{p} \gamma_{j} \cdot \frac{K\left(\Lambda_{j}\right)}{\operatorname{dim}\left(\Lambda_{j}\right)} .
\end{aligned}
$$

Here, $K\left(\Lambda_{j}\right)$ is the index of the representation $\rho_{j}: H_{j} \rightarrow$ Aut $\left(\Lambda_{j}\right)$. It is defined by $\operatorname{tr}\left(T \rho_{j}(x) T \rho_{j}(y)\right)=K\left(\Lambda_{j}\right) \cdot\langle x, y\rangle_{\mathfrak{h}_{j}}$ where $\langle,\rangle_{\mathfrak{h}_{j}}$ is the unique Ad-invariant metric on $\mathfrak{h}_{j}$ normalized so that the longest root has length squared equal to 2. The number $I\left(\mathfrak{h}_{j}\right)$ is the index of the embedding $H_{j} \rightarrow G$ defined by $\langle x, y\rangle_{\mathfrak{g}}=I\left(\mathfrak{h}_{j}\right) \cdot\langle x, y\rangle_{\mathfrak{h}_{j}}$ for $x, y \in \mathfrak{h}_{j}$. The number $L(G)$ is the length squared of the longest root of $G$, relative to the Killing metric. Tables of appropriate $L(G), I\left(\mathfrak{h}_{j}\right), K\left(\Lambda_{j}\right)$, and $\operatorname{dim}\left(\Lambda_{j}\right)$ can be found in [6,9]. In particular, Table II in [6] lists the $I\left(\mathfrak{h}_{j}\right)$ for the type I symmetric spaces. Four entries in that table listed as being greater than or equal to 1 are in fact equal to 1 and the misprinted value for $S O(5) / S O(3) \times S O(2)$ should be changed to 2 . Table 1 in this paper lists the values of the $\gamma_{j}$ constants for the type I symmetric spaces along with the isotropy representation $\mathfrak{m}$. The maximal weights describing representations depend on an ordering of simple roots and the ordering that is used appears in [4]. Finally, the values of the $\gamma_{0}$ 's were determined using the following generalization of Lemma 7.1 in [8].

Lemma 3.1. Let $G / H$ be a type I symmetric space with canonical decomposition $\mathfrak{g}=\mathfrak{h} \oplus \mathfrak{m}$. Let $V \subseteq \Lambda^{2} \mathfrak{m}$ be the kernel of the natural map $\Lambda^{2} \mathfrak{m} \rightarrow \mathfrak{h}$ which is induced by the alternating bilinear map $(x, y) \mapsto[x, y]: \mathfrak{m} \times \mathfrak{m} \rightarrow \mathfrak{h}$. Then although $V$ might be a reducible $H$-module, the Casimir operator of $V$ is exactly twice that of $\mathfrak{m}$, i.e., $\Gamma(V)=-1$.

Proof. The subspace $V$ is $H$-invariant because $\operatorname{Ad}_{h}[x, y]=\left[\operatorname{Ad}_{h} x, \operatorname{Ad}_{h} y\right]$ $\forall h \in H, x, y \in \mathfrak{m}$. Fix an orthonormal basis $\left\{e_{p}\right\}$ for $\mathfrak{g}$, relative to $-B_{\mathfrak{g}}$, so that the initial $e$ 's span $\mathfrak{h}$ and the remaining $e$ 's span $\mathfrak{m}$. Use subscripts $i, j, k, \ldots$ for $e$ 's in $\mathfrak{h}$ and subscripts $\alpha, \beta, \kappa, \lambda, \ldots$ for $e$ 's in $m$. In particular, this will be used for the summation convention. Define structure 
constants by $\left[e_{i}, e_{j}\right]=C_{i j}^{k} e_{k},\left[e_{i}, e_{\alpha}\right]=C_{i \alpha}^{\beta} e_{\beta}$, and $\left[e_{\alpha}, e_{\beta}\right]=C_{\alpha \beta}^{i} e_{i}$. Note, this final expression reflects the fact that for symmetric spaces $[\mathfrak{m}, \mathfrak{m}] \subseteq \mathfrak{h}$. The ad-invariance of the Killing form implies $C_{\alpha \beta}^{i}=C_{\beta i}^{\alpha}$ so that the structure constants have a cyclic symmetry along with the usual skew symmetry.

Suppose $a^{\alpha \beta} e_{\alpha} \wedge e_{\beta} \in V \subseteq \wedge^{2} \mathfrak{m}$ where the summation goes over all $\alpha$ and $\beta$ by assuming $a^{\alpha \beta}=-a^{\beta \alpha}$. The assumption that this be in $V$ gives $a^{\alpha \beta} C_{\alpha \beta}^{i} e_{i}=0$. It follows that

$$
\begin{aligned}
\sum( & \left.\left(e_{i}\right)\right)^{2}\left(a^{\alpha \beta} e_{\alpha} \wedge e_{\beta}\right) \\
& =\rho\left(e_{i}\right)\left(a^{\kappa \beta} C_{i \kappa}^{\alpha}+a^{\alpha \kappa} C_{i \kappa}^{\beta}\right)\left(e_{\alpha} \wedge e_{\beta}\right) \\
& =\left(a^{\kappa \beta} C_{i \kappa}^{\lambda} C_{i \lambda}^{\alpha}+a^{\lambda \kappa} C_{i \kappa}^{\beta} C_{i \lambda}^{\alpha}+a^{\kappa \lambda} C_{i \kappa}^{\alpha} C_{i \lambda}^{\beta}+a^{\alpha \kappa} C_{i \kappa}^{\lambda} C_{i \lambda}^{\beta}\right)\left(e_{\alpha} \wedge e_{\beta}\right) \\
& =2 \cdot \Gamma(\mathfrak{m}) \cdot\left(a^{\alpha \beta} e_{\alpha} \wedge e_{\beta}\right)+\left(a^{\kappa \lambda} C_{\alpha \beta}^{i} C_{\kappa \lambda}^{i}\right)\left(e_{\alpha} \wedge e_{\beta}\right) \\
& =2 \cdot \Gamma(\mathfrak{m}) \cdot\left(a^{\alpha \beta} e_{\alpha} \wedge e_{\beta}\right),
\end{aligned}
$$

where the third step follows from

$$
\left[e_{i},\left[e_{i}, e_{\alpha}\right]\right]=C_{i \alpha}^{\beta} C_{i \beta}^{\kappa} e_{\kappa}=\Gamma(\mathfrak{m}) \delta_{\alpha}^{\kappa} e_{\kappa}
$$

and the Jacobi identity

$$
C_{\beta \kappa}^{i} C_{\alpha i}^{\lambda}+C_{\kappa \alpha}^{i} C_{\beta i}^{\lambda}+C_{\alpha \beta}^{i} C_{\kappa i}^{\lambda}=0
$$

\section{HERMITIAN SYMMETRIC SPACES}

Suppose $G / H$ is a symmetric space with canonical decomposition $\mathfrak{g}=$ $\mathfrak{h} \oplus \mathfrak{m}$. There is a one-to-one correspondence between $G$-invariant almost complex structures on $G / H$ and real linear endomorphisms $J: \mathfrak{m} \rightarrow \mathfrak{m}$ satisfying $J^{2}=-1$ and $J\left(\operatorname{Ad}_{h} x\right)=\operatorname{Ad}_{h} J(x) \quad \forall h \in H, x \in \mathfrak{m}$. If in addition, $\mathfrak{m}$ has an $\operatorname{Ad}(H)$-invariant inner product that is Hermitian with respect to $J$, i.e., $\langle J x, J y\rangle=\langle x, y\rangle$, then $G / H$ is a Hermitian symmetric space [5].

One fact that is demonstrated clearly by Table 1 is that although the subspace $\mathfrak{m}$ is irreducible as a real $H$-module for the compact irreducible Riemannian symmetric spaces, it can split into two conjugate representations after complexification. This splitting occurs precisely when the real representation $\mathfrak{m}$ is of complex or, conceivably, quaternionic type [2]. In fact, the quaternionic case does not occur because the two parts of $\mathfrak{m}_{\mathbb{C}}$ are not equivalent complex representations. The real version of Schur's lemma shows that in such cases $\operatorname{Hom}_{H}^{\mathbb{R}}(\mathfrak{m}, \mathfrak{m}) \cong \mathbb{C}$. The endomorphism $J$ corresponding to $i \in \mathbb{C}$ defines an invariant almost complex structure on the space. Moreover, these symmetric spaces are in fact Hermitian symmetric [4].

Theorem 4.1. Suppose $G / H$ is a Hermitian symmetric space with $H$ compact, $G$ semisimple, and the action of $G$ on $G / H$ effective. Then

(i) The canonical connection is the unique invariant connection in the bundle $G \rightarrow G / H$.

(ii) The canonical connection is the unique invariant affine connection on $G / H$

(iii) If $\rho: H \rightarrow \operatorname{Aut}(V)$ is an irreducible complex representation then the associated bundle $G \times_{H}$ Aut $(V) \rightarrow G / H$ has a unique $G$-invariant connection. 
TABLE 1. Isotropy representations and coefficients of Casimir operators for the type I irreducible Riemannian symmetric spaces.

Hermitian symmetric spaces are marked by $*$.

\begin{tabular}{|c|c|c|c|}
\hline \multicolumn{2}{|c|}{ Symmetric Space } & $\mathbf{m}_{\mathbb{C}}$ & $\left\{\gamma_{j}\right\}$ \\
\hline * AI & $S U(2) / S O(2)$ & $(\beta) \oplus(-\beta)$ & $\frac{1}{2}$ \\
\hline & $S U(3) / S O(3)$ & $\left(4 \lambda_{1}\right)$ & $\frac{1}{8}$ \\
\hline & $S U(4) / S O(4)$ & $\left(2 \lambda_{1}, 2 \lambda_{1}\right)$ & $\frac{3}{16}, \frac{3}{16}$ \\
\hline & $S U(n) / S O(n)$ & $\left(2 \lambda_{1}\right)$ & $\frac{n-1}{8}$ \\
\hline AII & $S U(2 n) / S P(n)(n \geq 2)$ & $\left(\lambda_{2}\right)$ & $\frac{2 n+1}{4}$ \\
\hline * AIII & $S U(2) / S\left(U_{1} \times U_{1}\right)$ & $(\beta) \oplus(-\beta)$ & $\frac{1}{2}$ \\
\hline * & $S U(1+q) / S\left(U_{1} \times U_{q}\right)$ & $\left(\beta, \lambda_{q-1}\right) \oplus\left(-\beta, \lambda_{1}\right)$ & $\frac{1}{2 q}, \frac{q-1}{2}$ \\
\hline * & $S U(p+q) / S\left(U_{p} \times U_{q}\right)$ & $\left(\beta, \lambda_{1}, \lambda_{q-1}\right) \oplus\left(-\beta, \lambda_{p-1}, \lambda_{1}\right)$ & $\frac{1}{2 p q}, \frac{p^{2}-1}{2(p+q)}, \frac{q^{2}-1}{2(p+q)}$ \\
\hline * BDI & $S O(5) / S O(2) \times S O(3)$ & $\left(\beta, 2 \lambda_{1}\right) \oplus\left(-\beta, 2 \lambda_{1}\right)$ & $\frac{1}{6}, \frac{1}{4}$ \\
\hline * & $S O(6) / S O(2) \times S O(4)$ & $\left(\beta, \lambda_{1}, \lambda_{1}\right) \oplus\left(-\beta, \lambda_{1}, \lambda_{1}\right)$ & $\frac{1}{8}, \frac{3}{8}, \frac{3}{8}$ \\
\hline * & $S O(2+q) / S O(2) \times S O(q)$ & $\left(\beta, \lambda_{1}\right) \oplus\left(-\beta, \lambda_{1}\right)$ & $\frac{1}{2 q}, \frac{q-1}{4}$ \\
\hline & $S O(6) / S O(3) \times S O(3)$ & $\left(2 \lambda_{1}, 2 \lambda_{1}\right)$ & $\frac{3}{16}, \frac{3}{16}$ \\
\hline & $S O(7) / S O(3) \times S O(4)$ & $\left(2 \lambda_{1}, \lambda_{1}, \lambda_{1}\right)$ & $\frac{3}{20}, \frac{3}{10}, \frac{3}{10}$ \\
\hline & $S O(3+q) / S O(3) \times S O(q)$ & $\left(2 \lambda_{1}, \lambda_{1}\right)$ & $\frac{3}{4(q+1)}, \frac{q(q-1)}{4(q+1)}$ \\
\hline & $S O(8) / S O(4) \times S O(4)$ & $\left(\lambda_{1}, \lambda_{1}, \lambda_{1}, \lambda_{1}\right)$ & $\frac{1}{4}, \frac{1}{4}, \frac{1}{4}, \frac{1}{4}$ \\
\hline & $S O(4+q) / S O(4) \times S O(q)$ & $\left(\lambda_{1}, \lambda_{1}, \lambda_{1}\right)$ & $\frac{3}{2(q+2)}, \frac{3}{2(q+2)}, \frac{q(q-1)}{4(q+2)}$ \\
\hline & $S O(p+q) / \mathrm{SO}(p) \times S O(q)$ & $\left(\lambda_{1}, \lambda_{1}\right)$ & $\frac{p(p-1)}{4(p+q-2)}, \frac{q(q-1)}{4(p+q-2)}$ \\
\hline * BDII & $S O(3) / S O(2)$ & $(\beta) \oplus(-\beta)$ & $\frac{1}{2}$ \\
\hline & $S O(5) / S O(4)$ & $\left(\lambda_{1}, \lambda_{1}\right)$ & $\frac{1}{2}, \frac{1}{2}$ \\
\hline & $S O(n+1) / S O(n)$ & $\left(\lambda_{1}\right)$ & $\frac{n}{4}$ \\
\hline * CI & $S P(1) / U(1)$ & $(\beta) \oplus(-\beta)$ & $\frac{1}{2}$ \\
\hline * & $S P(n) / U(n)$ & $\left(\beta, 2 \lambda_{1}\right) \oplus\left(-\beta, 2 \lambda_{n-1}\right)$ & $\frac{1}{n(n+1)}, \frac{n-1}{4}$ \\
\hline CII & $S P(p+q) / S P(p) \times S P(q)$ & $\left(\lambda_{1}, \lambda_{1}\right)$ & $\frac{p(2 p+1)}{2(p+q+1)}, \frac{q(2 q+1)}{2(p+q+1)}$ \\
\hline * DIII & $S O(2 n) / U(n)(n \geq 3)$ & $\left(\beta, \lambda_{2}\right) \oplus\left(-\beta, \lambda_{n-2}\right)$ & $\frac{1}{n(n-1)}, \frac{n+1}{4}$ \\
\hline EI & $E_{6} / S P(4)$ & $\left(\lambda_{4}\right)$ & $\frac{3}{2}$ \\
\hline EII & $E_{6} / S U(2) \cdot S U(6)$ & $\left(\lambda_{1}, \lambda_{3}\right)$ & $\frac{1}{8}, \frac{35}{24}$ \\
\hline * EIII & $E_{6} / S O(2) \cdot \operatorname{Spin}(10)$ & $\left(\beta, \lambda_{4}\right) \oplus\left(-\beta, \lambda_{5}\right)$ & $\frac{1}{32}, \frac{15}{8}$ \\
\hline EIV & $E_{6} / F_{4}$ & $\left(\lambda_{4}\right)$ & $\frac{13}{6}$ \\
\hline $\mathbf{E V}$ & $E_{7} / S U(8)$ & $\left(\lambda_{4}\right)$ & $\frac{7}{4}$ \\
\hline EVI & $E_{7} / S U(2) \cdot S O(12)$ & $\left(\lambda_{1}, \lambda_{5}\right) \operatorname{or}\left(\lambda_{1}, \lambda_{6}\right)$ & $\frac{1}{12}, \frac{11}{6}$ \\
\hline * EVII & $E_{7} / S O(2) \cdot E_{6}$ & $\left(\beta, \lambda_{1}\right) \oplus\left(-\beta, \lambda_{6}\right)$ & $\frac{1}{54}, \frac{13}{6}$ \\
\hline EVIII & $E_{8} / S O(16)$ & $\left(\lambda_{7}\right) \operatorname{or}\left(\lambda_{8}\right)$ & 2 \\
\hline EIX & $E_{8} / S U(2) \cdot E_{7}$ & $\left(\lambda_{1}, \lambda_{7}\right)$ & $\frac{1}{20}, \frac{133}{60}$ \\
\hline FI & $F_{4} / S U(2) \cdot S P(3)$ & $\left(\lambda_{1}, \lambda_{3}\right)$ & $\frac{1}{6}, \frac{7}{6}$ \\
\hline FII & $F_{4} / \operatorname{Spin}(9)$ & $\left(\lambda_{4}\right)$ & 2 \\
\hline $\mathbf{G}$ & $G_{2} / S U(2) \times S U(2)$ & $\left(\lambda_{1}, 3 \lambda_{1}\right)$ & $\frac{3}{8}, \frac{1}{8}$ \\
\hline
\end{tabular}


Proof. Theorem XI.9.6 in [5] shows that under these conditions on $G / H$ there is an element $z_{0}$ in the center of $\mathfrak{h}$ such that the complex structure $J: \mathfrak{m} \rightarrow$ $\mathfrak{m}$ is given by $J(x)=\left[z_{0}, x\right] \quad \forall x \in \mathfrak{m}$. By Wang's theorem [11] invariant connections in the bundle $G \rightarrow G / H$ are given by linear mappings $\xi: \mathfrak{m} \rightarrow \mathfrak{h}$ satisfying $\xi\left(\operatorname{Ad}_{h} x\right)=\operatorname{Ad}_{h} \xi(x) \quad \forall x \in \mathfrak{m}, h \in H$. Infinitesimally, it must be true that $\xi[z, x]=[z, \xi(x)] \quad \forall x \in \mathfrak{m}, z \in \mathfrak{h}$. Letting $z=z_{0}$ gives

$$
\xi(x)=-\xi\left(J^{2} x\right)=-\xi\left[z_{0}, J x\right]=-\left[z_{0}, \xi(J x)\right]=0 \quad \forall x \in \mathfrak{m} .
$$

So $\xi=0$ and $\xi$ is the canonical connection.

For (ii), invariant affine connections are described by bilinear mappings $\eta: \mathfrak{m} \times \mathfrak{m} \rightarrow \mathfrak{m}$ satisfying $\eta\left(\operatorname{Ad}_{h} x, \operatorname{Ad}_{h} y\right)=\operatorname{Ad}_{h} \eta(x, y) \quad \forall x, y \in \mathfrak{m}, h \in H$. Infinitesimally, the requirement is

$$
[z, \eta(x, y)]=\eta([z, x], y)+\eta(x,[z, y]) \quad \forall x, y \in \mathfrak{m}, z \in \mathfrak{h} .
$$

Letting $z=z_{0}$ gives

$$
J \eta(x, y)=\eta(J x, y)+\eta(x, J y) \quad \forall x, y \in \mathfrak{m},
$$

or, by using this twice,

$$
-\eta(x, y)=-\eta(x, y)+2 \cdot \eta(J x, J y)-\eta(x, y) \quad \forall x, y \in \mathfrak{m} .
$$

Thus

$$
\eta(x, y)=2 \cdot \eta(J x, J y)=4 \cdot \eta\left(J^{2} x, J^{2} y\right)=4 \cdot \eta(x, y) \quad \forall x, y \in \mathfrak{m} .
$$

This shows $\eta=0$, i.e., $\eta$ is the canonical connection.

For (iii), Wang's theorem [11] shows that invariant connections in the associated bundle $G \times{ }_{H}$ Aut $(V) \rightarrow G / H$ are given by linear mappings $\xi: \mathfrak{m} \rightarrow$ End $(V)$ satisfying

$$
\xi\left(\operatorname{Ad}_{h} x\right)=\rho(h) \circ \xi(x) \circ \rho(h)^{-1} \quad \forall x \in \mathfrak{m}, h \in H,
$$

or infinitesimally,

$$
\xi([z, x])=T \rho(z) \circ \xi(x)-\xi(x) \circ T \rho(z) \quad \forall x \in \mathfrak{m}, z \in \mathfrak{h} .
$$

In particular,

$$
\begin{aligned}
\xi(x) & =-\xi\left(J^{2} x\right)=-\xi\left(\left[z_{0}, J x\right]\right) \\
& =-T \rho\left(z_{0}\right) \circ \xi(J x)+\xi(J x) \circ T \rho\left(z_{0}\right)=0
\end{aligned}
$$

because $T \rho\left(z_{0}\right)$ is in the center of $\operatorname{End}(V)$. To see this, let $\lambda$ be an eigenvalue of $T \rho\left(z_{0}\right)$ and $S_{\lambda}$ be the corresponding eigenspace. Then $S_{\lambda}$ is invariant under the action of $\mathfrak{h}$. Indeed, for $z \in \mathfrak{h}$ and $v \in S_{\lambda}$

$$
T \rho\left(z_{0}\right)(T \rho(z) v)=T \rho(z) T \rho\left(z_{0}\right) v+T \rho\left(\left[z_{0}, z\right]\right) v=\lambda T \rho(z) v
$$

because $z_{0}$ is in the center of $\mathfrak{h}$. By the irreducibility of the representation $\rho$, $S_{\lambda}=V$. So $T \rho\left(z_{0}\right)=\lambda I$ and $T \rho\left(z_{0}\right)$ is in the center of $\operatorname{End}(V)$.

\section{THE PROOF OF THEOREM 2.1}

The determination of all invariant affine connections on type I symmetric spaces goes as follows. First, by Theorem 4.1 the canonical connection is unique for Hermitian symmetric spaces. This can also be observed from the representation theory. The tensor product of the representations $(j \cdot \beta)$ and $(k \cdot \beta)$ of 
$S^{1}$ is the representation $((j+k) \cdot \beta)$. This shows that the central parts of the representations in $\mathfrak{m}_{\mathbb{C}} \otimes \mathfrak{m}_{\mathbb{C}}$ will always be of the form $(2 \cdot \beta),(-2 \cdot \beta)$, or $(0)$. These will never give intertwining maps $\mathfrak{m} \otimes \mathfrak{m} \rightarrow \mathfrak{m}$.

In the non-Hermitian cases, a case by case computation was used to determine the multiplicity of $\mathfrak{m}$ in $\mathfrak{m} \otimes \mathfrak{m}$. Key aspects of the computation involve the splitting of $\mathfrak{m} \otimes \mathfrak{m}$ into $S^{2} \mathfrak{m} \oplus \bigwedge^{2} \mathfrak{m}$, dimension counting, and Young tableaux [3]. The fact that the trivial representation appears exactly once (and in $S^{2} \mathfrak{m}$ ) was needed in the exceptional cases where dimension counting was used. In addition, Lemma 3.1 and the computations in Table 1 were particularly useful for determining the decomposition of $\Lambda^{2} \mathfrak{m}$. Of course, the representation whose maximal weight is twice that of $\mathfrak{m}$ is also known to appear in $\mathfrak{m} \otimes \mathfrak{m}$.

For example, in the case $\mathbf{A I}=S U(n) / S O(n)$ for $n \geq 5$, the subspace $\mathfrak{m}=$ $\left(2 \lambda_{1}\right)$. Young tableau computations give $\Lambda^{2} \mathfrak{m}=\left(2 \lambda_{1}+\lambda_{2}\right) \oplus\left(\lambda_{2}\right)$ and $S^{2} \mathfrak{m}=$ $\left(4 \lambda_{1}\right) \oplus\left(2 \lambda_{2}\right) \oplus\left(2 \lambda_{1}\right) \oplus(0)$. These need to be modified slightly in the cases $n=5$ and $n=6$. The copy of $\mathfrak{m}$ in $S^{2} \mathfrak{m}$ gives rise to a one-dimensional family of invariant affine connections.

The example EIV $=E_{6} / F_{4}$ also has a one-dimensional family of invariant affine connections. In this case, dimensional considerations and Casimir operators give $\mathfrak{m}=\left(\lambda_{4}\right), \bigwedge^{2} \mathfrak{m}=\left(\lambda_{3}\right) \oplus\left(\lambda_{1}\right)$, and $S^{2} \mathfrak{m}=\left(2 \lambda_{4}\right) \oplus\left(\lambda_{4}\right) \oplus(0)$. This was also verified by an explicit weight computation using [1]. The copy of $\mathfrak{m}$ in $S^{2} \mathfrak{m}$ gives the invariant affine connections. Note, this computation also shows that the corresponding map $\eta: \mathfrak{m} \times \mathfrak{m} \rightarrow \mathfrak{m}$ is symmetric.

A number of examples include a copy of $S U(2)$ or $S O(3)$ with the corresponding action on $\mathfrak{m}$ being given by the two-dimensional representation $\left(\lambda_{1}\right)$. Since $\left(\lambda_{1}\right) \otimes\left(\lambda_{1}\right)=\left(2 \lambda_{1}\right) \oplus(0)$, such examples never have nontrivial invariant affine connections.

The examples EVI $=E_{7} / S U(2) \cdot S O(12)$ and EVIII $=E_{8} / S O(16)$ seemed somewhat peculiar. There does not seem to be any reason why $\mathfrak{m}$ should be built out of one spinor representation as compared to the other $-\left(\lambda_{7}\right)$ vs. $\left(\lambda_{8}\right)$ in the case of EVIII. Known results about tensor products of spinor representations [3] show that $\mathfrak{m}$ does not appear in $\mathfrak{m} \otimes \mathfrak{m}$ in either case.

\section{NATURAL MAPPINGS OF INVARIANT AFFINE CONNECTIONS}

In [8] it was proven that bi-invariant affine connections on a Lie group $G$ can be described by bilinear mappings $\eta: \mathfrak{g} \times \mathfrak{g} \rightarrow \mathfrak{g}$ satisfying $\eta\left(\operatorname{Ad}_{g} x, \operatorname{Ad}_{g} y\right)=$ $\operatorname{Ad}_{g} \eta(x, y) \quad \forall x, y \in \mathfrak{g}, g \in G$. In particular, the Lie bracket gives such a mapping on an arbitrary Lie group. When $G$ is compact and simple all such $\eta$ are multiples of the Lie bracket except in the case of $S U(n)$ for $n \geq 3$. In the case of $S U(n)$, there is a two-dimensional family of such connections spanned by $\eta_{1}(x, y)=[x, y]$ and

$$
\eta_{2}(x, y)=i \cdot(x \cdot y+y \cdot x)-\frac{2}{n} \cdot \operatorname{tr}(x \cdot y) \cdot i I .
$$

In this section it will be proven that the new invariant affine connections on the spaces $S U(n) / S O(n)$ and $S U(2 n) / S P(n)$ are induced from the mapping $\eta_{2}$.

Theorem 6.1. If $(G, H)$ is a reductive pair with reductive decomposition $\mathfrak{g}=$ $\mathfrak{h} \oplus \mathfrak{m}$, then there exist mappings $i^{*}: \mathscr{A}_{G \times G}(\operatorname{Fr}(G)) \rightarrow \mathscr{A}_{H \times H}(F r(H))$ and $\pi_{*}$ : $\mathscr{A}_{G \times G}(F r(G)) \rightarrow \mathscr{A}_{G}(F r(G / H))$ defined by $\left(i^{*} \eta\right)(x, y)=(\eta(x, y))^{\mathfrak{h}} \quad \forall x, y \in$ $\mathfrak{h}$ and $\left(\pi_{*} \eta\right)(x, y)=(\eta(x, y))^{\mathfrak{m}} \quad \forall x, y \in \mathfrak{m}$. 
Proof. Since the inclusion maps $\mathfrak{h} \rightarrow \mathfrak{g}$ and $\mathfrak{m} \rightarrow \mathfrak{g}$ and projection maps $\mathfrak{g} \rightarrow \mathfrak{h}$ and $\mathfrak{g} \rightarrow \mathfrak{m}$ are all linear, the mappings $i^{*} \eta$ and $\pi_{*} \eta$ are clearly bilinear. By reductivity, they are also $H$-module maps and thus $\left(i^{*} \eta\right)\left(\operatorname{Ad}_{h} x, \operatorname{Ad}_{h} y\right)=$ $\operatorname{Ad}_{h}\left(i^{*} \eta\right)(x, y) \quad \forall x, y \in \mathfrak{h}$ and $\left(\pi_{*} \eta\right)\left(\operatorname{Ad}_{h} x, \operatorname{Ad}_{h} y\right)=\operatorname{Ad}_{h}\left(\pi_{*} \eta\right)(x, y)$ $\forall x, y \in \mathfrak{m}$.

In the case of type I symmetric spaces, the Lie bracket operation on $G$ never descends to give invariant affine connections on $G / H$. This is just the property $[\mathfrak{m}, \mathfrak{m}] \subseteq \mathfrak{h}$. However, the operation $\theta(x, y)=i \cdot(x \cdot y+y \cdot x)$ of $\mathfrak{u}(n)$ can be pushed from $U(n)$ to $S U(n)$, giving the mapping $\eta_{2}$, and then to $S U(n) / S O(n)$ and $S U(2 n) / S P(n)$ as described in Theorem 6.1. A straightforward calculation shows that the resulting image is nontrivial in all cases except for $S U(4) / S P(2)$. These are precisely the invariant affine connections in Theorem 2.1. Note, in both cases the image of $\eta_{2}$ restricted to $\mathfrak{m} \times \mathfrak{m}$ is automatically contained in $\mathfrak{m}$ - the projection back onto $\mathfrak{m}$ is not necessary. By comparison, in the case $S U(p+q) / S\left(U_{p} \times U_{q}\right)$ the restriction of $\eta_{2}$ to $\mathfrak{m} \times \mathfrak{m}$ has image contained in $\mathfrak{h}$ so that $\pi_{*} \eta_{2}=0$.

\section{REFERENCES}

1. M. R. Bremner, R. V. Moody, and J. Patera, Tables of dominant weight multiplicities for representations of simple Lie algebras, Marcel Dekker, New York, 1985.

2. T. Bröcker and T. tom Dieck, Representations of compact Lie groups, Springer-Verlag, New York, 1985.

3. M. Fischler, Young-tableau methods for Kronecker products of representations of the classical groups, J. Math. Phys. 22 (1981), 637-648.

4. S. Helgason, Differential geometry, Lie groups, and symmetric spaces, Academic Press, New York, 1978.

5. S. Kobayashi and K. Nomizu, Foundations of differential geometry, Interscience, New York, 1963, 1969.

6. H. T. Laquer, Stability properties of the Yang-Mills functional near the canonical connection, Michigan Math. J. 31 (1984), 139-159.

7. _ Invariant gauge automorphisms of homogeneous principal bundles, Geom. Dedicata 33 (1990), 27-35.

8. _ Invariant affine connections on Lie groups, Trans. Amer. Math. Soc. (to appear).

9. W. G. McKay and J. Patera, Tables of dimensions, indices, and branching rules for representations of simple Lie algebras, Marcel Dekker, New York, 1981.

10. K. Nomizu, Invariant affine connections on homogeneous spaces, Amer. J. Math. 76 (1954), 33-65.

11. H. C. Wang, On invariant connections over a principal fibre bundle, Nagoya Math. J. 13 (1958), 1-19.

Department of Mathematics, Idaho State University, Pocatello, Idaho 83209

E-mail address: laquerht@csc.isu.edu 